\title{
Complete Shape Metric and Geodesic
}

\author{
Jean-Paul Zolésio \\ CNRS and INRIA, INRIA, 2004 route des Lucioles, \\ BP 93, 06902 Sophia Antipolis Cedex, France \\ jean-paul.zolesio@sophia.inria.fr
}

\begin{abstract}
We develop the framework for moving domain and geometry under minimal regularity (of moving boundaries). This question arose in shape control analysis and non cylindrical PDE analysis. We apply here this setting to the morphic measure between shape or images. We consider both regular and non smooth situations and we derive complete shape metric space with characterization of geodesic as being solution to Euler fluid-like equation. By the way, this paper also addresses the variational formulation for solution to the coupled Eulertransport system involving only condition on the convected terms. The analysis relies on compactness results which are the parabolic version to the Helly compactness results for the $\mathrm{BV}$ embedding in the linear space of integrable functions. This new compactness result is delicate but supplies to the lack of convexity in the convection terms so that the vector speed associated with the optimal tube (or moving domain), here the shape geodesic, should not be curl-free so that the Euler equation does not reduce to a classical Hamilton-Jacobi one. For topological optimization this geodesic construction is developed by level set description of the tube, and numerical algorithms are in the next paper of this book.
\end{abstract}

\section{Introduction to Shape Metrics}

The shape analysis arose in the early 70's from structural mechanics. The problem was to find a best shape which would minimize the compliance (the work of external forces in some elasticity modeling). Later this problem extended to optimal control-like situation in which the criteria to be extremized with respect to a geometrical shape had a more general form which implied the study of the so called material and shape derivatives for the solution of a partial differential equation with boundary conditions on the unknown part of boundary [8[12 16]. Very soon the concepts of topology on general shape families were introduced. The easiest one was the metric induced by the characteristic function of the shape (in this case the shape is just defined up to a zero measure subset). Besides this the thinner one was the Courant metric, see the book [6], which consists, very roughly speaking, in minimizing ||$T-I_{d}\|+\| T^{-1}-I_{d} \|$ for each application mapping a domain $\Omega_{0}$ onto another $\Omega_{1}$, the minimum being taken over the family of such invertible mappings $T$. Indeed this metric is not known to be differentiable and is very difficult to be computed in this very abstract and non geometrical form. Also by the class of the regularity imposed to the mappings $T$ in the theory, it derives that the domains $\Omega_{i}, i=0,1$ should be homeomorphic to one another and then should have the same topology. The aim of this work is to relax this metric definition in order to solve these two difficulties (i.e. give a geometrical interpretation with computational

A. Korytowski et al. (Eds.): System Modeling and Optimization, IFIP AICT 312, pp. 144-166, 2009.

(c) IFIP International Federation for Information Processing 2009 
algorithms using level set techniques and extend the metric to a larger class of domains having different topologies) but also and mainly to construct the geodesics. This last issue turns to have several applications in any kind of large deformation process but also in image analysis. Through a Fully Eulerian equivalent definition we shall characterize the geodesic tube as being built by solutions to a coupled incompressible Euler flow-transport equation (in case of given volume constraint); meanwhile we furnish a full mathematical result for such variational solution to the incompressible Euler flow which turns to be a new result concerning Euler equation. The new metric we present here, which in some sense is an extension of the Courant metric, is based on two main considerations: Shapes (or geometry) are elements of some set, say $\mathscr{F}$, and we consider all connecting tubes in $\mathscr{F}$. Then the metric is built on the shortest such tube which furnishes the geodesic, solution to some differentiable variational problem. Also we shall derive complete metric spaces. The concept of geodesic for usual metrics such as Hausdorff distance, or $L^{1}$ metric on characteristic functions makes no sense as there is obviously no hope to derive any local uniqueness for a shortest path. Here also we still have none such result (nor local stability for the geodesic) but this challenging question is hopeful as been formulated in term of local uniqueness for flow Euler-like equation to which we can add any viscosity perturbation. This paper follows [24]23] and the book [13]. The connecting tube concept arose in moving domain analysis and non cylindrical PDE study in the 90's, for example in [3|7|9|12 19 4|2|11|10|14|21|17].

\section{Connecting Tubes}

We consider the time interval as being $I=[0,1]$ and $D$, a bounded domain in $R^{N}$ with smooth boundary. We consider the set of characteristic functions

$$
\mathscr{C}=\left\{\zeta=\zeta^{2} \in L^{1}(I \times D)\right\} .
$$

We consider the continuous elements

$$
\mathscr{C}^{0}=\mathscr{C} \cap C^{0}\left(I, L^{1}(D)\right) .
$$

Being given two measurable subsets $\Omega_{i} \subset D, i=0,1$, we consider the family of connecting tubes

$$
\mathscr{T}^{0}\left(\Omega_{0}, \Omega_{1}\right)=\left\{\zeta \in \mathscr{C}^{0} \text { s.t. } \zeta(i)=\chi_{\Omega_{i}}, i=0,1\right\}
$$

\subsection{Moving Domain}

For any $\zeta \in \mathscr{T}^{0}\left(\Omega_{0}, \Omega_{1}\right)$ we consider the set $Q=\cup_{0<t<1}\{t\} \times \Omega_{t} \subset R^{N+1}$ such that $\zeta=\chi_{Q}$. This set $Q$ is defined up to an $N+1$ dimensional zero measure set.

\subsection{Generic Framework for Metric}

The idea for constructing metrics is to consider in this set the infimum of some norm for the time derivative term $\frac{\partial}{\partial t} \zeta$. Indeed if such term is zero then $\zeta$ is not time depending. The general setting is to consider families of admissible tubes such that $\frac{\partial}{\partial t} \zeta \in$ 
$L^{p}(I, \mathscr{H}(D))$ for some Banach space $\mathscr{H}(D)$ of distributions over $D, \mathscr{H}(D) \subset \mathscr{D}^{\prime}(D)$, and consider the following connecting tubes:

$$
\mathscr{T}_{\mathscr{H}}^{0, p}\left(\Omega_{0}, \Omega_{1}\right)=\left\{\zeta \in \mathscr{T}^{0}\left(\Omega_{0}, \Omega_{1}\right) \text {, s.t. } \frac{\partial}{\partial t} \zeta \in L^{p}(I, \mathscr{H}(D))\right\},
$$

and for some $p \geq 1$, the metric in the following form:

$$
d_{p, \mathscr{H}}\left(\Omega_{0}, \Omega_{1}\right)=\operatorname{Inf}_{\zeta \in \mathscr{T}_{\mathscr{H}}^{0, p}\left(\Omega_{0}, \Omega_{1}\right)} \int_{0}^{1}\left\|\frac{\partial}{\partial t} \zeta(t)\right\|_{\mathscr{H}(D)}^{p} d t .
$$

\subsection{The Time $L^{p}$ Regularity of $\frac{\partial}{\partial t} \zeta$ Implies $\zeta \in \mathscr{C}^{0}$}

Let us define

$$
\mathscr{C}_{\mathscr{H}}^{0, p}=\left\{\zeta \in \mathscr{C} \text { s.t. } \frac{\partial}{\partial t} \zeta \in L^{p}(I, \mathscr{H}(D))\right\}
$$

Proposition 1. Let $p \geq 1$, then $\mathscr{C}_{\mathscr{H}}^{0, p} \subset \mathscr{C}^{0}$.

Proof. Obviously we have

$$
\mathscr{C}_{\mathscr{H}}^{0, p} \subset W^{1, p}(I, \mathscr{H}(D)) \subset C^{0}(I, \mathscr{H}(D)) \subset C^{0}\left(I, \mathscr{D}^{\prime}(D)\right)
$$

So that from the following Lemma we get $\mathscr{C}_{\mathscr{H}}^{0, p} \subset C^{0}\left(I, L^{1}(D)\right)$; then we see that the continuity property of the tube derives directly from $\zeta \in \mathscr{C}$ (that is $\zeta=\zeta^{2}$ ) and the weak regularity of the time derivative measure $\frac{\partial}{\partial t} \zeta$.

Lemma 1. Let $\zeta=\zeta^{2} \in L^{1}(I \times D) \cap C^{0}\left(I, \mathscr{D}^{\prime}(D)\right)$. Then $\zeta \in C^{0}\left(I, L^{1}(D)\right)$.

Proof. Notice that

$$
\|\zeta(t+s)-\zeta(t)\|_{L^{1}(D)}=\|\zeta(t+s)-\zeta(t)\|_{L^{2}(D)}^{2} .
$$

Then it is enough to show that $\zeta \in C^{0}\left(I, L^{2}(D)\right)$. We begin by establishing the weak $L^{2}(D)$ continuity: for any element $f \in L^{2}(D)$ consider

$$
\begin{aligned}
\int_{D}(\zeta(t+s)(x)-\zeta(t)(x)) f(x) d x & =\int_{D}(\zeta(t+s, x)-\zeta(t, x)) \phi(x) d x \\
& +\int_{D}(\zeta(t+s, x)-\zeta(t, x))(f(x)-\phi(x)) d x .
\end{aligned}
$$

Let be given $\varepsilon>0$, by the choice of $\phi \in \mathscr{D}^{\prime}(D)$ (using here the density of $\mathscr{D}^{\prime}(D)$ in $\left.L^{2}(D)\right)$, we have

$$
\left|\int_{D}(\zeta(t+s, x)-\zeta(t, x))(f(x)-\phi(x)) d x\right| \leq \int_{D}|f(x)-\phi(x)| d x \leq \varepsilon .
$$

So we derive the continuity for the weak $L^{2}(D)$ topology. To reach the strong topology it is sufficient now to consider the continuity of the mapping

$$
t \rightarrow \int_{D}|\zeta(t, x)|^{2} d x=\int_{D} \zeta(t, x) d x=((\zeta(t), 1))_{L^{2}(D)} .
$$




\subsection{Metric and Pseudo Metric}

We consider a set $\bar{\Omega} \subset D$ and the family of all subsets in $D$ which are reachable in finite time from this $\bar{\Omega}$ by elements $\zeta, \zeta$ describing the whole set $\mathscr{C}_{\mathscr{H}}^{0, p}$; more precisely:

$$
\mathscr{O}_{\bar{\Omega}}=\left\{\Omega \subset D \text { s.t. } \exists \zeta \in \mathscr{C}_{\mathscr{H}}^{0, p} \text { with } \chi_{\Omega}=\zeta(1), \chi_{\bar{\Omega}}=\zeta(0)\right\} .
$$

Notice that by construction any pair of elements in this family is connected:

$$
\forall\left(\Omega_{0}, \Omega_{1}\right) \in\left(\mathscr{O}_{\bar{\Omega}}\right)^{2} \text {, the family } \mathscr{T}_{\mathscr{H}}^{0, p}\left(\Omega_{0}, \Omega_{1}\right) \text { is not empty. }
$$

Proposition 2. For any $p \geq 1, d_{p, \mathscr{H}}$ is a quasi-metric in the following sense; for any elements $\Omega_{i}, i=0,1,2$ in $\mathscr{O}_{\bar{\Omega}}$ we have:

i) $d_{p, \mathscr{H}}\left(\Omega_{0}, \Omega_{1}\right)=0$ iff $\chi_{\Omega_{0}}=\chi_{\Omega_{1}}$,

ii) $d_{p, \mathscr{H}}\left(\Omega_{0}, \Omega_{1}\right)=d_{p, \mathscr{H}}\left(\Omega_{1}, \Omega_{0}\right)$,

iii) $d_{p, \mathscr{H}}\left(\Omega_{0}, \Omega_{2}\right) \leq 2^{p-1}\left(d_{p, \mathscr{H}}\left(\Omega_{0}, \Omega_{1}\right)+d_{p, \mathscr{H}}\left(\Omega_{1}, \Omega_{2}\right)\right)$.

For $p=1, d_{1, \mathscr{H}}$ is a metric on $\mathscr{O}_{\bar{\Omega}}$.

Proof. 1a) When $\chi_{\Omega_{1}}=\chi_{\Omega_{2}}$ as elements in $L^{1}(D)$, we may choose $\zeta(t, x)=\chi_{\Omega_{0}}(x)$ so that the evolution domain $Q$ is the cylinder $Q=I \times \Omega_{0}$ and $\frac{\partial}{\partial t} \zeta=0$ realizes the minimum and leads to the null distance.

1b) Conversely for any $\varepsilon>0$ there exists some admissible tube $\zeta^{\varepsilon}$ with $\zeta^{\varepsilon}(i)=\chi_{\Omega_{i}}$ and realizing the infimum up to $\varepsilon$. Then

$$
\left\|\zeta^{\varepsilon}(0)-\zeta^{\varepsilon}(1)\right\|_{\mathscr{H}(D)} \leq \int_{0}^{1}\left\|\frac{\partial}{\partial t} \zeta^{\varepsilon}\right\|_{\mathscr{H}} d t \leq\left(\int_{0}^{1}\left\|\frac{\partial}{\partial t} \zeta^{\varepsilon}\right\|_{\mathscr{H}}^{p} d t\right)^{1 / p} \leq \varepsilon^{1 / p} .
$$

We conclude $\chi_{\Omega_{1}}=\chi_{\Omega_{0}}$ as elements in $\mathscr{H}(D)$.

2) The symmetry is obviously realized by reversing the time variable. Indeed if $\zeta^{\varepsilon}$ realizes the infimum up to $\varepsilon$ then we consider $\tilde{\zeta}^{\varepsilon}(t, x):=\zeta^{\varepsilon}(1-t, x)$ and $\int_{0}^{1}\left\|\frac{\partial}{\partial t} \tilde{\zeta}^{\varepsilon}\right\|_{\mathscr{H}}^{p} d t=$ $\int_{0}^{1}\left\|\frac{\partial}{\partial t} \zeta^{\varepsilon}\right\|_{\mathscr{H}}^{p} d t$ so that the element $\tilde{\zeta}^{\varepsilon}$ also approaches the infimum up to $\varepsilon$.

3) The triangle property derives from the following obvious generic construction: let us consider two connecting tubes $\zeta^{k, \varepsilon} \in \mathscr{T}_{\mathscr{H}(D)}^{0, p}\left(\Omega_{k-1}, \Omega_{k}\right), k=1,2$ and realizing the infimum up to $\varepsilon$ in the corresponding distances $d_{\mathscr{H}(D)}\left(\Omega_{k-1}, \Omega_{k}\right)$.

We introduce the new element $\bar{\zeta}^{\varepsilon} \in \mathscr{T}_{\mathscr{H}(D)}^{0, p}\left(\Omega_{0}, \Omega_{1}\right)$ piecewisely defined as follows:

$$
\begin{aligned}
& \forall t \in[0,1 / 2], \bar{\zeta}^{\varepsilon}(t)=\zeta^{1, \varepsilon}(2 t) ; \forall t \in[1 / 2,1], \bar{\zeta}^{\varepsilon}(t)=\zeta^{2, \varepsilon}(2 t-1) \\
& \int_{0}^{1}\left\|\frac{\partial}{\bar{\partial} t} \bar{\zeta}^{\varepsilon}\right\|_{\mathscr{H}}^{p} d t=\int_{0}^{1 / 2}\left\|\frac{\partial}{\partial t} \bar{\zeta}^{\varepsilon}\right\|_{\mathscr{H}}^{p}+\int_{1 / 2}^{1}\left\|\frac{\partial}{\partial t} \bar{\zeta}^{\varepsilon}\right\|_{\mathscr{H}}^{p} d t .
\end{aligned}
$$

Now

$$
\forall t \in[0,1 / 2], \frac{\partial}{\partial t} \bar{\zeta}^{\varepsilon}(t)=2 \frac{\partial}{\partial t} \zeta^{1, \varepsilon}(2 t) ; \forall t \in[1 / 2,1], \frac{\partial}{\partial t} \bar{\zeta}^{\varepsilon}(t)=2 \frac{\partial}{\partial t} \zeta^{2, \varepsilon}(2 t-1) .
$$

Then

$$
\int_{0}^{1}\left\|\frac{\partial}{\bar{\partial} t} \bar{\zeta}^{\varepsilon}\right\|_{\mathscr{H}}^{p} d t=2^{p} \int_{0}^{1 / 2}\left\|\frac{\partial}{\partial t} \zeta^{1, \varepsilon}(2 t)\right\|_{\mathscr{H}}^{p} d t+2^{p} \int_{1 / 2}^{1}\left\|\frac{\partial}{\partial t} \zeta^{2, \varepsilon}(2 t-1)\right\|_{\mathscr{H}}^{p} d t .
$$


By respective changes of variables $s=2 t$ and $s=2 t-1$ we get

$$
\int_{0}^{1}\left\|\frac{\partial}{\bar{\partial} t} \bar{\zeta} \varepsilon\right\|_{\mathscr{H}}^{p} d t=2^{p-1} \int_{0}^{1}\left\|\frac{\partial}{\partial t} \zeta^{1, \varepsilon}(s)\right\|_{\mathscr{H}}^{p} d s+2^{p-1} \int_{0}^{1}\left\|\frac{\partial}{\partial t} \zeta^{2, \varepsilon}(s)\right\|_{\mathscr{H}}^{p} d s .
$$

So that $\forall \varepsilon>0$ we have:

$$
d_{p, \mathscr{H}}\left(\Omega_{0}, \Omega_{2}\right) \leq \int_{0}^{1}\left\|\frac{\partial}{\bar{\partial} t} \bar{\zeta}^{\varepsilon}\right\|_{\mathscr{H}}^{p} d t \leq 2^{p-1}\left(d_{p, \mathscr{H}}\left(\Omega_{0}, \Omega_{1}\right)+d_{p, \mathscr{H}}\left(\Omega_{1}, \Omega_{2}\right)+2 \varepsilon\right) .
$$

\subsection{Banach Space of Bounded Measures}

We make the choice, as Banach space of measures $\mathscr{H}(D)$, of the space of bounded measure $M^{1}(D)$ and set

$$
p \geq 1, \mathscr{C}^{p}=\left\{\zeta \in \mathscr{C} \text { s.t. } \frac{\partial}{\partial t} \zeta \in L^{p}\left(I, M^{1}(D)\right)\right\}
$$

that is

$$
\mathscr{C}^{p}=\mathscr{C} \cap L^{p}(I, B V(D)) .
$$

From the previous considerations we get $\mathscr{C}^{p} \subset C^{0}\left(I, L^{1}(D)\right)$, so that

$$
p \geq 1, \mathscr{C}^{p}=\left\{\zeta \in \mathscr{C}^{0} \text { s.t. } \frac{\partial}{\partial t} \zeta \in L^{p}\left(I, M^{1}(D)\right)\right\} .
$$

The set of connecting tubes is then:

$$
\mathscr{T}^{p}\left(\Omega_{0}, \Omega_{1}\right)=\left\{\zeta \in \mathscr{C}^{p} \text { s.t. } \zeta(i)=\chi_{\Omega_{i}}, i=0,1\right\} .
$$

Corollary 1. Let $p \geq 1$, then

$$
d_{p}\left(\Omega_{0}, \Omega_{1}\right)=\operatorname{Inf}_{\zeta \in \mathscr{T}^{p}\left(\Omega_{0}, \Omega_{1}\right)} \int_{0}^{1}\left\|\frac{\partial}{\partial t} \zeta(t)\right\|_{M^{1}(D)}^{p} d t
$$

is a quasi metric. When $p=1, d_{1}$ is a metric.

\subsection{Smooth Domains}

When a tube $\zeta=\chi_{Q}$ is smooth, $Q=\cup_{0<t<1}\{t\} \times \Omega_{t}$, with lateral boundary $\Sigma=$ $\cup_{0<t<1}\{t\} \times \partial \Omega_{t}$ being a $C^{k}$ manifold in $I \times D \subset R^{N+1}$, with the integer $k \geq 1$, there exists a vector field $V \in C^{0}\left(\bar{I}, C^{k}\left(D, R^{N}\right)\right)$ with $\left\langle V(t, x), n_{\partial D}>=0\right.$ such that $\Omega_{t}$ is built by the flow mapping of $V$, that is $\Omega_{t}=T_{t}(V)\left(\Omega_{0}\right)$.

For example, when $k=2$ the oriented distance function $b_{\Omega_{t}}=d_{\Omega_{t}}-d_{\Omega_{t}^{c}} \in C^{2}(\mathscr{U})$ where $\mathscr{U}$ is some tubular neighborhood of the boundary $\partial \Omega_{t}$, and we may choose any extension of $\nabla b_{\Omega_{t}}(x) v(t) o p_{t}(x)$ as speed vector $V(t, x)$, where the normal field is $n_{t}(x)=\nabla b_{\Omega_{t}}(x), x \in \Gamma_{t}=\partial \Omega_{t}$, the projection $p_{t}$ onto $\Gamma_{t}$ being defined in $\mathscr{U}$ by $p_{t}(y)=$ $y-b_{\Omega_{t}}(y) \nabla b_{\Omega_{t}}(y)$ (we recall that $\nabla b_{\Omega_{t}} o p_{t}(y)=\nabla b_{\Omega_{t}}(y)$ for any $y \in \mathscr{U}$ ). 
In the smooth situation the tube characteristic function $\zeta$ verifies the classical convection problem (in weak sense):

$$
\zeta^{2}=\zeta \in L^{1}(I \times D), \frac{\partial}{\partial t} \zeta+\nabla \zeta \cdot V=0, \zeta(0)=\chi_{\Omega_{0}} .
$$

Then, without any restriction, we consider smooth domains generated from $\Omega_{0}$ by the flow mapping $T_{t}(V)$ of smooth vector fields $V(t, x), V \in E^{k}$ with:

$$
E^{k}:=\left\{V \in C^{0}\left(\bar{I}, C^{k}\left(\bar{D}, R^{N}\right)\right) \text { s.t. } \forall t \in \bar{I},\left\langle V(t), n_{\partial D}\right\rangle=0\right\} .
$$

The connecting condition is then: $\Omega_{1}=T_{1}(V)\left(\Omega_{0}\right)$, where $T_{t}(V)$ is the flow mapping of $V$ at time $t \in[0,1]$. We set $\Omega_{t}:=T_{t}(V)\left(\Omega_{0}\right)$ and $\zeta(t,)=.\chi_{\Omega_{t}}$ is an admissible connecting tube, moreover we have:

$$
\left\|\frac{\partial}{\partial t} \zeta(t)\right\|_{M^{1}(D)}=\int_{\partial \Omega_{t}}\left|\langle V(t, x)), n_{t}(x)\right\rangle \mid d \Gamma_{t}(x)
$$

and the metric would turn to be

$$
d_{k, p}\left(\Omega_{0}, \Omega_{1}\right)=\operatorname{Inf}_{V \in \mathscr{V} k}\left(\Omega_{0}, \Omega_{1}\right) \int_{0}^{1}\left(\int_{\partial \Omega_{t}(V)}\left|\left\langle V(t, x), n_{t}(x)\right\rangle\right| d \Gamma_{t}(x)\right)^{p} d t
$$

where $\mathscr{V}_{k}\left(\Omega_{0}, \Omega_{1}\right)$, defined below, stands for the family of connecting vector fields in $E^{k}, k \geq 1$. As the time regularity required for the classical flow analysis is just time continuity (in the very definition of $E^{k}$ ) this connecting family turns to be stable through the generic construction of connecting vector field $\bar{V}$ similar to the point 3 in the proof of Proposition 2.

Proposition 3. Let $k \geq 1$ and $\bar{\Omega}$ be open domain in $D \subset R^{N}$ whose boundary $\bar{\Gamma}$ is a $C^{k}$ manifold. We consider the family of smooth domains

$$
\mathscr{O}_{k}=\left\{\Omega \subset D \text { s.t. } \exists V \in E^{k}, \Omega=T_{1}(V)(\bar{\Omega})\right\} .
$$

For any pair of elements $\Omega_{i}, i=0,1$ in this family, the set of connecting fields

$$
\mathscr{V}_{k}:=\left\{V \in E^{k} \text { s.t. } T_{1}(V)\left(\Omega_{0}\right)=\Omega_{1}\right\}
$$

is never empty. Equipped with $d_{p, k}$, the family $\mathscr{O}_{k}$ is a p-quasi-metric space (and a metric space when $p=1$ ).

An important point here is that in this family $\mathscr{O}_{k}, k \geq 1$, all domains are homeomorphic to the domains $\bar{\Omega}$ so that we cannot evaluate distance between domains with different topologies, even when they are smooth. In order to escape from that classical difficulty we shall develop two classes of issues. The first one is based on time piecewise regularity of domains leading to a good modeling for classical topological changes such as holes collapse or holes creation (at a given time $t_{0}$ ), and topological separations. The second one is based on completing different approach relying on the fully eulerian description of tubes with non smooth vector fields $V$. 


\subsubsection{The Piecewise Smooth Situation}

In some applications we shall consider the situation in which the time interval can be decomposed in a finite number of time intervals of smoothness for the lateral boundaries: we consider tubes such that there exists an integer $K$ (tube dependent) and time partitions $t_{k}$ such that

$$
\left.I=\cup_{1 \leq k \leq K} \bar{I}_{k}, I_{k}=\right] t_{k-1}, t_{k}[.
$$

We assume that for $t \in I_{k}$ the lateral boundary $\Sigma_{k}$ of the set $Q_{k}=\cup_{t \in I_{k}}\{t\} \times \Omega_{t}, \Sigma_{k}=$ $\cup_{t \in I_{k}}\{t\} \times \partial \Omega_{t}$, is a $C^{1}$ manifold in $R^{N+1}$. We consider the unit normal field $v_{k}$ to $\Sigma_{k}$, out going to $Q_{k}$ on $\Sigma_{k}$. It can be uniquely written in the form

$$
\forall t \in I_{k}, \forall x \in \Gamma_{k}, v_{k}(t, x)=\frac{1}{\sqrt{1+v_{k}(t, x)^{2}}}\left(-v_{k}(t, x), n_{t}(x)\right) \in R_{t} \times R_{x}^{N} .
$$

The term $v_{k}(t,$.$) is called the normal speed of the moving boundary \Gamma_{t}$. Obviously we have

$$
\forall t \in I_{k}, \forall \phi \in \mathscr{D}(D),\left\langle\frac{\partial}{\partial t} \zeta, \phi\right\rangle=\int_{\Gamma_{t}} v_{k}(t, x) \phi(x) d \Gamma_{k}(x) .
$$

\subsubsection{Behavior of the Normal Speed at $t=t_{i}$}

To discuss the global regularity of $\frac{\partial}{\partial t} \zeta$ we must choose the regularity of $v$ at the junction times $t_{k}$. Consider

$$
\begin{aligned}
\left\langle\zeta,-\frac{\partial}{\partial t} \Phi\right\rangle_{L^{p}\left(I, L^{2}(D)\right) \times L^{q}(I)} & =\int_{0}^{1} \int_{\Omega_{t}}-\frac{\partial}{\partial t} \Phi(t, x) d t d x \\
& =\Sigma_{k=1, \ldots, K} \int_{t_{k-1}}^{t_{k}} \int_{\Omega_{t}}-\frac{\partial}{\partial t} \Phi(t, x) d t d x
\end{aligned}
$$

But

$$
\forall t \in I_{k}, \frac{\partial}{\partial t} \int_{\Omega_{t}} \Phi(t, x) d t d x=\int_{\Gamma_{t}^{k}} \Phi(t, x) v_{k}(t, x) d \Gamma_{t}^{k}(x)+\int_{\Omega_{t}} \frac{\partial}{\partial t} \Phi(t, x) d x .
$$

So that assume that $v_{k} \in L^{1}\left(\Sigma_{k}\right)$ and as $\zeta \in C^{0}\left(I, L^{1}(D)\right)$ we have

$$
\begin{aligned}
& \int_{t_{k-1}+\varepsilon}^{t_{k}-\varepsilon} \int_{\Omega_{t}} \frac{\partial}{\partial t} \Phi(t, x) d t d x=\int_{t_{k-1}+\varepsilon}^{t_{k}-\varepsilon} \int_{\Gamma_{t}}\left|v_{k}(t, x)\right| d \Gamma_{k}(x) d t \\
& \quad+\int_{D}\left(\zeta\left(t_{k-1}+\varepsilon, x\right) \Phi\left(t_{k-1}+\varepsilon, x\right)-\zeta\left(t_{k}-\varepsilon, x\right) \Phi\left(t_{k}-\varepsilon, x\right)\right) d x \\
& \left.\rightarrow{ }_{\varepsilon \rightarrow 0} \int_{t_{k-1}}^{t_{k}} \int_{\Gamma_{t}}\left|v_{k}(t, x)\right| d \Gamma_{k}(x) d t+\int_{D}\left(\zeta\left(t_{k-1}, x\right) \Phi\left(t_{k-1}, x\right)-\zeta\left(t_{k}, x\right)\right) \Phi\left(t_{k}, x\right)\right) d x
\end{aligned}
$$

Finally we get $\forall \Phi \in \mathscr{D}(I \times D)$,

$$
\begin{aligned}
\left\langle\zeta, \frac{\partial}{\partial t} \Phi\right\rangle & =\lim _{\mathcal{E} \rightarrow 0} \Sigma_{1 \leq k \leq K} \int_{t_{k-1}+\varepsilon}^{t_{k}-\varepsilon} \int_{D} \zeta(t, x) \Phi(t, x) d x \\
& =\int_{0}^{1} \int_{\partial \Omega_{t}} v(t, x) \Phi(t, x) d \Gamma_{t}(x) d x
\end{aligned}
$$


This expression continuously extends for any $\Phi \in C_{c}^{0}(I \times D)$ (with compact support) and we get

$$
\left\|\frac{\partial}{\partial t} \zeta(t)\right\|_{L^{1}\left(I, M^{1}(D)\right)}=\int_{0}^{1} \int_{\Gamma_{t}}|v(t, x)| d \Gamma_{t}(x),
$$

and we have

$$
\int_{0}^{1}\left\|\frac{\partial}{\partial t} \zeta(t)\right\|_{M^{1}(D)}^{p} d t=\int_{0}^{1}\left(\int_{\Gamma_{t}}|v(t, x)| d \Gamma_{t}(x)\right)^{p} d t .
$$

\subsection{3 "Piecewise Metric"}

Proposition 4. Let $\bar{\Omega}$ be a smooth subset in $D, k \geq 1, p \geq 1$. We consider the family $\mathscr{O}_{\text {pwk }}(\bar{\Omega})$ of all subsets connected to $\bar{\Omega}$ by piecewise $C^{k}$ tubes in the previous sense and verifying the following qualification condition:

$$
\int_{0}^{1} \int_{\Gamma_{t}}|v(t, x)| d \Gamma_{t}(x) d t<\infty .
$$

Then equipped with

$$
\delta_{p w k}^{p}\left(\Omega_{0}, \Omega_{1}\right)=\operatorname{Inf}_{\zeta \in \mathscr{T}_{p w k}^{p}\left(\Omega_{0}, \Omega_{1}\right)} \int_{0}^{1}\left(\int_{\Gamma_{t}}|v(t, x)| d \Gamma_{t}(x)\right)^{p} d t,
$$

the family $\mathscr{O}_{p w k}^{p}(\bar{\Omega})$ is a $p$-quasi-metric space. For $p=1$, the family $\mathscr{O}_{p w k}^{1}(\bar{\Omega})$ equipped with $\delta_{p w k}^{1}$, is a metric space.

Notice that a sufficient condition for deriving the condition (40) is that the lateral surface $\Sigma$ would have a finite $\mathscr{H}^{n-1}$ Hausdorff measure (that is to say that the tube $Q$ has a finite perimeter in $I \times D)$. Indeed we have:

$$
P_{I \times D}(Q)=\int_{0}^{1} \int_{\Gamma_{t}} \sqrt{1+v^{2}} d \Gamma_{t}(x) d t \geq \int_{0}^{1} \int_{\Gamma_{t}}|v| d \Gamma_{t}(x) d t .
$$

\subsubsection{Level Set Formulation}

Let $\Psi(t, x) \in C^{1}(\bar{I} \times \bar{D})$ and consider

$$
\forall t \in I, \Omega_{t}=\{x \in D \text { s.t. } \Psi(t, x)>0\}, \Gamma_{t}=\{x \in D \text { s.t. } \Psi(t, x)=0\} .
$$

An important case is when the function has the following form

$$
\Psi(t, x)=\Phi(x)-t \text { then } \Omega_{t}=\{x \in D \text { s.t. } \Phi(x)>t\} .
$$

In this very situation, from Sard's theorem we know that for almost every $t$ in $I$ the manifold $\Gamma_{t}$ is of class $C^{1}$ which does not insure the tube associated to $\Psi$ to be pwk (even for $k=1$ ).

In the general setting the qualification condition (40) would write

$$
\int_{0}^{1} \int_{\Gamma_{t}}\left(\frac{\left|\frac{\partial}{\partial t} \Psi\right|}{\left\|\nabla_{x} \Psi\right\|}\right)(t, x) d \Gamma_{t}(x)<\infty .
$$


We shall restrict our study to the pwk level set tubes, i.e. functions $\Pi(t, x)$ such that the generated tubes verify the previous pw 1 condition: $\exists t_{k}, t_{0}=0<t_{1}<\ldots<t_{K}=1 \mathrm{such}$ that on each open interval $\left.I_{k}=\right] t_{k-1}, t_{K}[$,

$$
\exists \alpha_{k}(.) \in C^{0}\left(I_{k}\right) \text { s.t. } \forall x \in \Gamma_{t},\left\|\nabla_{x} \Psi(t, x)\right\| \geq \alpha_{k}(t)>0 .
$$

In this class the previous piecewise tubes analysis applies and we get an associated metric in terms of level sets. In the proof of the following result the only main point is to verify that in the generic construction for the triangle axiom (point 3 in the proof of Proposition 2 ) the connecting element $\bar{\zeta} \varepsilon$ piecewisely defined is still in the class. Indeed $\bar{\zeta} \varepsilon$ is associated to the function

$$
\bar{\Psi}^{\varepsilon}(t, x)= \begin{cases}\Psi^{1, \varepsilon}(2 t, x), & \text { if } 0<t<1 / 2 \\ \Psi^{2, \varepsilon}(2 t-1, x), & \text { if } 1 / 2<t<1\end{cases}
$$

Obviously this element $\bar{\Psi}^{\varepsilon}$ verifies the two conditions (45) and 46) if the element $\zeta^{i, \varepsilon}, i=0,1$ does.

Proposition 5. Let $\bar{\Omega} \subset D$ be a $C^{1}$ domain. We consider the family

$$
\begin{aligned}
& \mathscr{P}_{p w 1}=\left\{\Psi \in C^{1}(\bar{I} \times \bar{D}), \text { s.t. } \exists t_{k}, 1 \leq k \leq K_{\Psi},\right. \text { s.t. } \\
& \Sigma_{k}=\left\{(t, x) \mid t \in I_{k}, \Psi(t, x)=0\right\} \\
& \text { is a } \left.C^{1} \text { manifold in } R^{N+1} \text { and } \Psi \text { verifying (45), (46) }\right\} .
\end{aligned}
$$

We also consider the family generated by this class of piecewise $C^{1}(p w 1)$ functions:

$$
\mathscr{O}_{L S}=\left\{\Omega=\{x \in D \mid \Psi(1, x)>0\}, \Psi \in \mathscr{P}_{p w 1}\right\} .
$$

Obviously two elements $\Omega_{i}, i=0,1$ in this family are connected by tube in the form of (47) and we denote

$$
\mathscr{T}_{L S}\left(\Omega_{0}, \Omega_{1}\right)=\left\{\Psi \in \mathscr{P}_{p w 1} \text { s.t. } \Omega_{i}=\{x \in D \mid \Psi(i, x)>0\}\right\} .
$$

We set

$$
\delta_{L S}\left(\Omega_{0}, \Omega_{1}\right)=\operatorname{Inf} \Psi_{\Psi \in \mathscr{T}_{L S}\left(\Omega_{0}, \Omega_{1}\right)} \int_{0}^{1} \int_{\Psi(t)^{-1}(0)}\left|\frac{\partial}{\partial t} \Psi(t, x)\right|\left\|\nabla_{x} \Psi(t, x)\right\|^{-1} d \Gamma_{t}(x) d t .
$$

Then equipped with $\delta_{L S}$ the family $\mathscr{O}_{L S}$ is a metric space.

\subsubsection{Submetrics}

In the level set setting it is easy to describe some connecting elements. Assume that $\Omega_{i}=\left\{x \in D \mid \Phi_{i}(x)>0\right\}, i=0,1$. Then let

$$
\Psi(t, x)=\rho(t) \Phi_{1}(x)+(1-\rho(t)) \Phi_{0}(x), \text { with } \rho \in C^{1}(\bar{I}), \rho(i)=i, i=0,1,
$$

and we could consider the "submetric" associated to these connections, for different admissible such functions $\rho$. 


\subsubsection{Level Set Metric Associated to Subspace}

In the definition (48) of the set of "potential" functions $\Psi$ we can limit to a given subspace of functions in the following way: let $E$ be a closed subspace in $C^{1}(\bar{I} \times \bar{D})$, we consider

$$
\mathscr{P}_{p w E}=\mathscr{P}_{p w 1} \cap E
$$

As $\mathscr{P}_{p w E} \subset \mathscr{P}_{p w 1}$ we get the similar inclusions $\mathscr{O}_{L S E} \subset \mathscr{O}_{S L}, \mathscr{T}_{L S E}\left(\Omega_{0}, \Omega_{1}\right) \subset \mathscr{T}_{L S}$ $\left(\Omega_{0}, \Omega_{1}\right)$ and the family $\mathscr{O}_{L S E}$ is equipped with the metric $\delta_{L S E} \leq \delta_{L S}$.

In the specific situation where the Banach space is of finite dimension we consider the Galerkin-like construction. Let $E_{1}, \ldots . . E_{M}$ be $M$ given elements in $C^{1}(\bar{D})$ and consider

$$
E=\left\{e(t, x)=\Sigma_{1 \leq m \leq M} \lambda_{m}(t) E_{m}(x) \mid \lambda \in C^{1}(I)^{M}\right\} .
$$

When the elements $E_{m}(x)$ are chosen as polynomial functions the surfaces $\Gamma_{t}$ are algebraic surfaces (or curves) in $D$ and it is an open question to characterize conditions on the coefficients $\lambda$ in order that the tube satisfies (45) and (46). Nevertheless in applications it seems difficult to violate them.

\section{Complete Metric: Existence of Geodesic}

We address now the question concerning the infima in the previous metrics (or pseudo metrics) we described in the previous sections. Let $\zeta^{n}$ be a minimizing sequence in (24). The element $\frac{\partial}{\partial t} \zeta^{n}$ remains bounded in $L^{p}\left(I, M^{1}(D)\right)$. Then when $p>1$, there exists a subsequence, still denoted $\frac{\partial}{\partial t} \zeta^{n}$ and weakly converging to an element $\omega \in$ $L^{p}\left(I, M^{1}(D)\right)$. The difficulty is now to get $\omega$ in the form $\omega=\frac{\partial}{\partial t} \zeta^{*}$ for some admissible $\zeta^{*}$. As $\zeta^{n} \in \mathscr{C}^{0}$, it remains bounded as an element of $\mathscr{C}$ in $L^{r}(I \times D)$, and this for any $1 \leq r \leq \infty$. Let us consider a subsequence $\zeta^{n}$ weakly convergent to some element $\rho$. By continuity of the derivative in weak topologies we derive that $\omega=\frac{\partial}{\partial t} \rho$ but a priori the element $\rho$ is not a characteristic function. Indeed we shall have $\rho \in \mathscr{C}$, that is $\rho^{2}=\rho$, if and only if $\rho^{n}$ strongly converges to $\rho$ in $L^{1}(I \times D)$. Nevertheless, this strong $L^{1}(I \times D)$ convergence would not imply the limiting element $\rho$ to be in $\mathscr{C}^{0}$. Now, if this element is not time continuous the connection makes no sense and it could not be a candidate for geodesic.

\subsection{Compacity Arguments and Complete Metric}

\subsubsection{Surface Tension-Like Term}

We shall propose now several changes in the metric (or $p$-quasi-metric) to derive this strong convergence. First of all let us denote that if we complete in (24) the metric by the following, with $\sigma>0$ (a surface tension term)

$$
d\left(\Omega_{0}, \Omega_{1}\right)=\operatorname{Inf}_{\zeta \in \mathscr{T}\left(\Omega_{0}, \Omega_{1}\right)} L_{\sigma}(\zeta)
$$

with

$$
L_{\sigma}=\int_{0}^{1}\left\|\frac{\partial}{\partial t} \zeta\right\|_{M^{1}(D)} d t+\sigma \int_{0}^{1}\left\|\nabla_{x} \zeta(t)\right\|_{M^{1}\left(D, R^{N}\right)} d t,
$$

then we could derive, for any smooth minimizing sequence, $\zeta^{n}(t, x)=\chi_{Q^{n}}$, the tubes with bounded perimeter in $I \times D$ as we have. 
3.1.2 Boundedness of the Perimeter in $I \times D \subset R^{N+1}$

Proposition 6. Assume the evolution domain $Q$ to be smooth, then

$$
P_{I \times D}(Q) \leq \int_{0}^{1}\left\|\frac{\partial}{\partial t} \zeta\right\|_{M^{1}(D)} d t+\int_{0}^{1}\left\|\nabla_{x} \zeta(t)\right\|_{M^{1}\left(D, R^{N}\right)} d t
$$

Proof

$$
\begin{aligned}
& P_{I \times D}(Q)=\int_{\Sigma} d \Sigma=\int_{0}^{1} \int_{\Gamma_{t}} \sqrt{1+v^{2}} d \Gamma_{t}(x) d t \\
& P_{I \times D}(Q) \leq \int_{0}^{1} \int_{\Gamma_{t}}(1+|v|) d \Gamma_{t}(x) d t=\int_{0}^{1}\left(P_{D}\left(\Omega_{t}\right)+\int_{\Gamma_{t}}|v| d \Gamma_{t}\right) d t
\end{aligned}
$$

but

$$
P_{D}\left(\Omega_{t}\right)=\|\nabla \zeta(t)\|_{M^{1}\left(D, R^{N}\right)}, \int_{\Gamma_{t}}|v| d \Gamma_{t}(x)=\left\|\frac{\partial}{\partial t} \zeta\right\|_{M^{1}(D)} .
$$

So that 57 is true when the domain is smooth.

\subsubsection{Metric on the Closure of Smooth Tubes Would Fail}

We could hope that (57), by some density arguments, extends for all tubes $\zeta \in L^{1}$ $(I, B V(D)) \cap W^{1,1}\left(I, M^{1}(D)\right)$ (which is an open question) or define the metric as follows. Introducing the family of smooth tubes, say $\mathscr{C}^{\infty}$ (elements $\zeta=\chi_{Q}$ with lateral boundary being a $C^{\infty}$ manifold in $I \times D \subset R^{N+1}$ ), set

$$
d_{\sigma}^{\infty}\left(\Omega_{0}, \Omega_{1}\right)=\operatorname{Inf}_{\zeta \in \mathscr{C}^{\infty}} L_{\sigma}(\zeta)
$$

Any minimizing sequence, from (57) would remain bounded in $B V(I \times D)$ and then there shall exist a subsequence strongly converging in $L^{1}(I \times D)$ so that the limiting element will be $\zeta \in \mathscr{C}$ with

$$
\|\zeta\|_{B V(I \times D)} \leq \operatorname{limin} f_{n \rightarrow \infty}\left\|\zeta_{n}\right\|_{B V(I \times D)}
$$

and by similar weak 1.s.c. arguments on each term of $L_{\sigma}$ we would see that the limiting element $\zeta \in \mathscr{C}$ would be a minimizer of $L_{\sigma}$ on some closure of $\mathscr{C}^{\infty}$. Nevertheless this element would not belong to $\mathscr{C}^{0}$, and being not continuous in time the connection property $\zeta \in \mathscr{T}\left(\Omega_{0}, \Omega_{1}\right)$ could be lost and this candidate for metric would fail, while having a minimizer. Finally we understand that even if the inequality (57) extends to a more general family of tubes it would not help for deriving metric with geodesic.

An important point here is that any expression in the form of

$$
\tilde{d}_{\sigma}\left(\Omega_{0}, \Omega_{1}\right)=\operatorname{Inf}_{\zeta \in \mathscr{T}} L_{\sigma}(\zeta)
$$

would fail to be a metric because it violates the first metric axiom. Indeed the new perimeter term $\sigma \int_{0}^{1} P_{D}\left(\Omega_{t}\right) d t$ cannot be zero. 


\subsection{Compactness Results}

We have seen that the compactness result deriving from the boundedness of $L_{\sigma}$, i.e. boundedness in the Banach space

$$
\mathscr{B}^{1}=L^{1}(I, B V(D)) \cap W^{1,1}\left(I, M^{1}(D)\right) \subset C\left(I, M^{1}(D)\right)
$$

is not enough.

Proposition 7. Consider $\zeta_{n}$ bounded in $L^{1}(I, B V(D))$, together with $\frac{\partial}{\partial t} \zeta_{n}$ bounded in $L^{p}\left(I, M^{1}(D)\right)$ for some $p>1$. Then there exists a subsequence, still denoted $\zeta_{n}$, and an element $\zeta \in L^{1}(I, B V(D)) \cap W^{1,1}\left(I, M^{1}(D)\right) \subset C^{0}\left(I, M^{1}(D)\right)$ such that $\zeta_{n}$ strongly converges to $\zeta$ in $L^{1}\left(I, L^{1}(D)\right)$ with $\frac{\partial}{\partial t} \zeta \in L^{p}\left(I, M^{1}(D)\right)$ verifying $\|\zeta\|_{L^{1}(I, B V(D))} \leq$ $\liminf \left\|\zeta_{n}\right\|_{L^{1}(I, B V(D))}$ and $\left\|\frac{\partial}{\partial t} \zeta\right\|_{L^{p}\left(I, M^{1}(D)\right)} \leq \liminf \left\|\frac{\partial}{\partial t} \zeta_{n}\right\|_{L^{p}\left(I, M^{1}(D)\right)}$.

Continuity $\zeta \in W^{1,1}\left(I, M^{1}(D)\right)$ implies $\zeta \in C^{0}\left(I, L^{1}(D)\right)$. Moreover $\zeta(t, x)=\zeta^{2}(t, x)$, a.e. $(t, x) \in I \times D$ and $\zeta \in C^{0}\left(I, L^{1}(D)\right)$ implies that the mapping:

$$
t \in \bar{I} \rightarrow p(t):=\left\|\nabla_{x} \zeta(t)\right\|_{M^{1}\left(D, R^{N}\right)} \text { is s.c.i. }
$$

Proof. See [24[13].

Also a similar compacity result can be derived with $p=1$, leading to a metric, but assuming some uniform integrability for the $\left\|\frac{\partial}{\partial t} \zeta\right\|_{M^{1}(D)}$ term.

Proposition 8. Consider $\zeta_{n}$ bounded in $L^{1}(I, B V(D))$ together with $\frac{\partial}{\partial t} \zeta_{n}$ bounded in $L^{1}\left(I, M^{1}(D)\right)$, and assume there exists an element $\theta \in L^{1}(I)$ such that

$$
\text { a.e. } t \in I,\left\|\frac{\partial}{\partial t} \zeta_{n}\right\|_{M^{1}(D)} \leq \theta(t) \text {. }
$$

Then there exists a subsequence, still denoted $\zeta_{n}$, and an element $\zeta \in L^{1}(I, B V(D)) \cap$ $W^{1,1}\left(I, M^{1}(D)\right) \subset C^{0}\left(I, M^{1}(D)\right)$ such that $\zeta_{n}$ strongly converges to $\zeta$ in $L^{1}\left(I, L^{1}(D)\right)$ with $\frac{\partial}{\partial t} \zeta \in L^{p}\left(I, M^{1}(D)\right)$ verifying $\|\zeta\|_{L^{1}(I, B V(D))} \leq$ liminf $\left\|\zeta_{n}\right\|_{L^{1}(I, B V(D))}$ and $\left\|\frac{\partial}{\partial t} \zeta\right\|_{L^{p}\left(I, M^{1}(D)\right)} \leq \liminf \left\|\frac{\partial}{\partial t} \zeta_{n}\right\|_{L^{p}\left(I, M^{1}(D)\right)}$.

Continuity $\zeta \in W^{1,1}\left(I, M^{1}(D)\right)$ implies $\zeta \in C^{0}\left(I, L^{1}(D)\right)$. Moreover $\zeta(t, x)=\zeta^{2}(t, x)$, a.e. $(t, x) \in I \times D$ and $\zeta \in C^{0}\left(I, L^{1}(D)\right)$ implies that the mapping:

$$
t \in \bar{I} \rightarrow p(t):=\left\|\nabla_{x} \zeta(t)\right\|_{M^{1}\left(D, R^{N}\right)} \text { is s.c.i. }
$$

Proof. See [21|13].

\subsection{Use of Compactness}

The idea is to consider the following expression for the shape metric defined by 24b:

$$
\bar{d}^{p}\left(\Omega_{0}, \Omega_{1}\right)=\operatorname{Inf}_{\zeta \in \mathscr{T}^{p}\left(\Omega_{0}, \Omega_{1}\right)} \int_{0}^{1}\left\|\frac{\partial}{\partial t} \zeta(t)\right\|_{M^{1}(D)} d t+" \int_{0}^{1}\left|p_{\zeta}^{\prime}(t)\right|^{p} d t^{\prime} .
$$


Indeed the last term is not finite in general as it would imply $p(t)$ to be time continuous which is known to be false (the perimeter is l.s.c. only and may "jump down" as in the celebrate "Camembert entamé" example: take a circular cheese Camembert with radius $R$ and subtract a radial triangular part with angle $\alpha$, the perimeter is $p(\alpha)$ and $\left.\operatorname{limin} f_{\alpha \rightarrow 0} p(\alpha)=(2 \pi+2) R>p(0)=2 \pi R\right)$.

We relax this term by introducing (see [23]) the "time capacity" term

$$
\theta^{p}(\zeta)=\operatorname{Inf}_{\mu \in K^{p}(\zeta)} \int_{0}^{1}\left|\mu^{\prime}(t)\right|^{p} d t
$$

with the closed convex set

$$
K^{p}(\zeta)=\left\{\mu \in W^{1, p}(I) \text { s.t. }\left\|\nabla_{x} \zeta(t)\right\|_{M^{1}\left(D, R^{N}\right)} \leq \mu(t) \text { a.e. } t \in I\right\} .
$$

Then the metric is

$$
\bar{d}^{p}\left(\Omega_{0}, \Omega_{1}\right):=\operatorname{Inf}_{\zeta \in \mathscr{T} p\left(\Omega_{0}, \Omega_{1}\right)} \int_{0}^{1}\left\|\frac{\partial}{\partial t} \zeta(t)\right\|_{M^{1}(D)} d t+\theta^{p}(\zeta) .
$$

\subsection{Complete Quasi-Metric by Level Set Formulation}

Let $p>1$ and $\Omega_{i}, i=1,2$ be two arbitrary measurable subsets in $D$. Let

$$
\begin{aligned}
K\left(\Omega_{1}, \Omega_{2}\right)=\left\{\phi \in L^{2}\left(I, H^{1}(D)\right) \cap W^{1,1}\left(I, L^{2}(D)\right),\right. & \\
& \frac{\partial}{\partial t} \phi \in L^{p}\left(I, L^{2}(D)\right), \\
& \left.\Omega_{1}=\{\Phi(0, .)>0\}, \Omega_{2}=\{\Phi(1, .)>0\}\right\} .
\end{aligned}
$$

Notice that $K\left(\Omega_{1}, \Omega_{2}\right) \subset C^{0}\left(\bar{I}, L^{2}(D)\right)$. We set

$$
d_{L S, p}\left(\Omega_{1}, \Omega_{2}\right):=\operatorname{Inf}_{\phi \in K\left(\Omega_{1}, \Omega_{2}\right)} \int_{0}^{1}\left(\alpha\|\phi(t)\|_{H^{1}(D)}^{2}+\left\|\frac{\partial}{\partial t} \phi(t)\right\|_{L^{2}(D)}^{p}\right) d t .
$$

Proposition 9. Let $1<p \leq 2$. Equipped with $d_{L S, p}$, the family of measurable subsets in $D$ is a complete quasi-metric space.

\section{Fully Eulerian Metric $d_{e}$}

For non smooth vector fields, being given the element $\Omega_{0}$ in $D$ the problem 25 may have no solution or several solutions (depending on the weak regularity of the speed vector field $V$ ). As soon as $V$ satisfies the minimal regularity $V \in \mathscr{V}^{p}$ where

$$
\begin{gathered}
p \geq 1, \mathscr{V}^{p}=\left\{V \in L^{p}\left(I \times D, R^{N}\right), \operatorname{div} V \in L^{p}(I \times D),\right. \\
\left.\left\langle V, n_{\partial D}\right\rangle=0 \text { in } W^{-1, p}(\partial D)\right\},
\end{gathered}
$$

the following classical convection problem

$$
\zeta \in L^{1}(I \times D), \frac{\partial}{\partial t} \zeta+\nabla \zeta . V=0, \zeta(0)=\chi_{\Omega_{0}}
$$


possesses solution (the proof is classically done by the Galerkin method when $V \in$ $L^{2}(I \times D)$ and $(\operatorname{div} V)^{+} \in L^{\infty}\left(I, L^{2}(D)\right)$, see [20], and there is no uniqueness result for the solution, which a priori is not an element in $\mathscr{C}$, nor in $\mathscr{C}^{0}$ ). The element $\zeta$ is not a characteristic function but is time continuous, $\zeta \in C^{0}\left([0,1], H^{-1 / 2}(D)\right)$. Indeed we consider weak solutions to problems (25) and (74), in the following sense:

$$
\begin{gathered}
\forall \phi \in C^{\infty}(\bar{I} \times \bar{D}) \text { s.t. } \phi(1, .)=0 \\
\int_{0}^{1} \int_{D} \zeta\left(-\frac{\partial}{\partial t} \phi-\operatorname{div}(\phi V)\right) d x d t=\int_{D} \chi_{\Omega_{0}} \phi(0, x) d x .
\end{gathered}
$$

The time derivative, for any solution to (74) (then to 25 ) verifies:

$$
\frac{\partial}{\partial t} \zeta=\operatorname{div}(\zeta V)-\zeta \operatorname{div} V \in L^{p}\left(I, W^{-1, p}(D)\right),
$$

so that

$$
\zeta \in W^{1, p}\left(I, W^{-1, p}(D)\right) \subset C^{0}\left(\bar{I}, W^{-1, p}(D)\right) .
$$

Notice that weak solutions to (74) can also be obtained by the following technique, without any $L^{\infty}$ requirement on the divergence:

Proposition 10. Let $p>1$ and $V \in \mathscr{V}^{p}$ defined in (73), let $\left.V_{n} \in \mathscr{V}^{p} \cap C^{\infty}\left(\bar{I} \times \bar{D}, R^{N}\right)\right)$ such that $V_{n} \rightarrow V$ strongly in $\mathscr{V}^{p}$. Consider the element $\zeta_{n}(t)=\chi_{\Omega_{0}} o T_{t}\left(V_{n}\right)^{-1} \in \mathscr{C}^{0}$, a unique solution to the characteristic convection problem (25). There exists a subsequence, still denoted $\zeta_{n}$ which weakly converges in $L^{p}(I \times D)$ to an element $\rho \in$ $L^{p}(I \times D) \cap W^{1,1}\left(I, W^{-1, p}(D)\right) \subset C^{0}\left(\bar{I}, W^{-1, p}(D)\right)$, a solution to the convection problem (74) or (75).

Proof. We pass to the limit in the weak form (75).

The concept of distance between the two sets $\Omega_{i}, i=0,1$ is associated to the "shortest path", that is now introduced through the Euler description using the product space approach which is described in [23] and [24]. Let us consider the eulerian connecting tubes defined as set of couples $(\zeta, V)$ solving the convection equation:

$$
\mathscr{T}_{e}^{p}\left(\Omega_{0}, \Omega_{1}\right)=\left\{(\zeta, V) \in \mathscr{C} \times \mathscr{V}^{p} \text { solving (25) with } \zeta(i)=\chi_{\Omega_{i}}, i=0,1\right\} .
$$

\subsection{Eulerian Metrics}

Let

$$
d_{p}^{e}\left(\Omega_{0}, \Omega_{1}\right):=\operatorname{Inf}_{(\zeta, V) \in \mathscr{T}^{p}\left(\Omega_{0}, \Omega_{1}\right)} \int_{0}^{1}\left(\|\left. V(t)\right|_{L^{p}\left(D, R^{N}\right)} ^{p}+|\operatorname{div} V(t)|_{L^{p}(D)}^{p}\right) d t
$$

and

$$
\bar{d}_{e}^{p}\left(\Omega_{0}, \Omega_{1}\right):=\operatorname{Inf}_{(\zeta, V) \in \mathscr{T}_{e}^{p}\left(\Omega_{0}, \Omega_{1}\right)} \int_{0}^{1} \int_{D}\left(|V|^{p}+(\operatorname{div} V)^{p}\right) d x d t+\theta^{p}(\zeta) .
$$


Proposition 11. For $p \geq 1, d_{p}^{e}$ is a quasi-metric in the following sense:

i) $d_{p}^{e}\left(\Omega_{0}, \Omega_{1}\right)=0$ iff $\chi_{\Omega_{0}}=\chi_{\Omega_{1}}$,

ii) $d_{p}^{e}\left(\Omega_{0}, \Omega_{1}\right)=d_{p}^{e}\left(\Omega_{1}, \Omega_{0}\right)$,

iii) $d_{p}^{e}\left(\Omega_{0}, \Omega_{2}\right) \leq 2^{p-1}\left(d_{p}^{e}\left(\Omega_{0}, \Omega_{1}\right)+d_{p}^{e}\left(\Omega_{1}, \Omega_{2}\right)\right)$.

Moreover, equipped with $\bar{d}_{p}^{e}$ the family $\mathscr{O}_{\bar{\Omega}}^{e}$ is a complete quasi-metric space and for $p=1$, equipped with $d_{1}^{e}$, it is a complete metric space.

\section{2 $B V$ Regularity of the Field $V$}

When the speed vector field $V$ verifies some BV properties, $V \in L^{2}\left(I, B V(D)^{N}\right)$ ([12324]), there is a unique tube associated to $V$, then we do have an application $V \rightarrow \zeta_{V}$ and with such regularity on $V$ we can revisit the complete metric $d$ being completely delivered of the non differential perimeter and curvature terms that we were obliged to introduce in order to apply the compacity theorems. From the tube analysis we consider several interesting choices for the space regularity of the speed vector field (together with its divergence field). Let

$$
\mathscr{E}^{1,1}=\left\{V \in L^{1}\left(I \times D, R^{N}\right) \text { s.t. } \operatorname{div} V \in L^{1}(D), V . n_{D} \in W^{-1,1}(\partial D)\right\},
$$

and let $E$ be a closed subspace in $B V(D) \cap \mathscr{E} 1,1$ such that any element $V \in E$ verifies the required assumptions. A first example is, when working with prescribed volume for the moving domain

$$
E_{0}=\left\{V \in B V\left(D, R^{N}\right) \cap \mathscr{E}^{1,1} \text {, s.t. } \operatorname{div} V=0 \text { a.e. }(t, x) \in I \times D\right\}
$$

$V$ be a divergence-free vector field with $\operatorname{div} V=0, V \in L^{1}\left(I, E_{0}\right)$, where $E=B V\left(D, R^{N}\right)$ or any closed subspace (for example $E=\left\{V \in H_{0}^{1}\left(D, R^{N}\right)\right.$, s.t. $\left.\operatorname{div} V=0\right\}$ ). An obvious metric is to consider the set

$$
\begin{gathered}
\mathscr{V}\left(\Omega_{1}, \Omega_{2}\right)=\left\{V \in \mathscr{E}^{1,1} \text { s.t. } V, \operatorname{div} V \in L^{p}\left(I, E_{0}\right), \text { s.t. } \zeta_{0}=\chi_{\Omega_{1}}, \zeta(1)=\chi_{\Omega_{2}}\right\} \\
\delta_{E_{0}}\left(\Omega_{1}, \Omega_{2}\right)=\operatorname{Inf}_{V \in \mathscr{V}\left(\Omega_{1}, \Omega_{2}\right)} \int_{0}^{1}\|V(t)\|_{E_{0}} d t .
\end{gathered}
$$

As $V$ is divergence-free the previous boundedness assumption on the divergence is verified and to each $V$ a tube $\zeta_{V}$ is associated through the convection. Then we get:

Proposition 12. Let $E$ be any subspace of $B V\left(D, R^{N}\right) \cap \mathscr{E}^{1,1}$ such that any element $V$ satisfies assumptions of Theorem 2.12 of [24], for example $E=E_{0}$. Then equipped with $\delta_{E}$, the family $\mathscr{O}_{\Omega_{0}}^{E}$ is a metric space.

$$
p>1, d_{E_{0}}\left(\Omega_{1}, \Omega_{2}\right)=\operatorname{Inf}_{V \in \mathscr{V}\left(\Omega_{1}, \Omega_{2}\right)}\|V\|_{L^{p}\left(I, E_{0}\right)}+\left\|\frac{\partial}{\partial t} V\right\|_{L^{1}\left(I, M^{1}\left(D, R^{N}\right)\right)} .
$$

Proposition 13. Let $E$ be any subspace of $B V\left(D, R^{N}\right) \cap \mathscr{E}^{1,1}$, such that any element $V$ whose divergence satisfies assumptions of Theorem 2.12 of [24]. Then equipped with $d_{E}$ the family $\mathscr{O}_{\Omega_{0}}^{E}$ is a complete quasi-metric space. 


\subsubsection{Geodesic Characterization via Transverse Field $Z$}

In that setting we are concerned with smooth vector fields $Z(s, t, x) \in R^{N}$ such that $Z(s, 0, x)=Z(s, 1, x)=0$ so that the extremities (for $t=0, t=1$ ) of the perturbed tube $Q_{s}:=T_{s}(Z(s))(Q)$ are preserved. The parameter $s$ appears here as a perturbation parameter. Indeed the family of connecting tubes $\mathscr{T}_{e}^{p}\left(\Omega_{0}, \Omega_{1}\right)$ is not a linear space nor equipped with any manifold structure. Nevertheless we can describe some tangential space $\mathbf{T}_{(\zeta, V)} \mathscr{T}_{e}^{p}\left(\Omega_{0}, \Omega_{1}\right)$ at any element (tube) $(\zeta, V)$ : if $(\zeta, V) \in \mathscr{T}$ then $\left.\zeta o T_{s}(Z(t))^{-1}, V^{s}\right) \in \mathscr{T}$ where $\left[\frac{d}{d s} V^{s}\right]_{s-0}=Z_{t}+[Z, V]$. The previous study for the transverse field [18]13[11] implies that for given such a vector field $Z$, with $\operatorname{div}_{x} Z(s, t, x)=0$ we get the admissible perturbation of the field $V$ in the form $V+s W(s, t, x)$, with

$$
W(s, t, x)=\frac{\partial}{\partial t} Z(s, t, x)+[Z, V] .
$$

More precisely, define the Lipschitz-continuous connecting set:

$$
\mathscr{V}^{1, \infty}\left(\Omega_{1}, \Omega_{2}\right)=\left\{V \in L^{1}\left(I, W^{1, \infty}\right) \cap \mathscr{E}^{1,1}, \text { s.t. } \zeta_{V} \in \overline{\mathscr{T}}\left(\Omega_{1}, \Omega_{2}\right)\right\}
$$

and the set of smooth transverse vector fields:

$$
\mathscr{Z}=\left\{Z(t, x) \in C_{\text {comp }}^{\infty}\left(I \times D, R^{N}\right)\right\} .
$$

Notice that $\operatorname{such} Z$ verifies $Z(0,)=.Z(1,)=$.0 on $D$.

Proposition 14. Let $V \in \mathscr{V}\left(\Omega_{1}, \Omega_{2}\right)$ and $Z(t, x) \in \mathscr{Z}$. The transformation $\mathscr{T}=$ $T_{s}(Z) o T_{t}(V)$ maps $\Omega_{t}(V)$ onto $\Omega_{t}^{s}:=T_{s}(Z)\left(\Omega_{t}(V)\right)$ so that

$$
\begin{aligned}
\forall s, \forall Z, V^{s}(t, x) & =\frac{\partial}{\partial t} \mathscr{T}_{o} \mathscr{T}^{-1} \\
& =\left(\frac{\partial}{\partial t} T_{s}(Z(t))+D T_{s}(Z(t)) . V(t)\right) o T_{s}(Z(t))^{-1} \in \mathscr{V}^{1, \infty}\left(\Omega_{1}, \Omega_{2}\right) .
\end{aligned}
$$

Lemma 2

$$
\left.\frac{\partial}{\partial s} V^{s}(t, x)\right|_{s=0}=\frac{\partial}{\partial t} Z(t)+[Z(t), V(t)] .
$$

Corollary 2. Consider a functional $\mathscr{J}(V)=j\left(\zeta_{V}\right)$ and let $\bar{V}$ be a minimizing element of $\mathscr{J}$ on $\mathscr{V}\left(\Omega_{1}, \Omega_{2}\right)$. Then we have

$$
\forall Z \in \mathscr{Z}, \frac{\partial}{\partial s} \mathscr{J}\left(\bar{V}^{s}\right)_{s=0}=J^{\prime}\left(\bar{V} ;\left(\frac{\partial}{\partial s} V^{s}\right)_{s=0}\right)=\mathscr{J}^{\prime}\left(\bar{V} ; \frac{\partial}{\partial t} Z(t)+[Z(t), V(t)]\right) \geq 0 .
$$

That variational principle extends to vector field $V \in E$ for which the flow mapping $T_{t}(V)$ is poorly defined. The element $\zeta_{V} \in \mathscr{H}^{c}$ is uniquely defined. For any $Z \in \mathscr{Z}$ the perturbed $\zeta_{V}^{s}:=\zeta_{V} o T_{s}(Z)^{-1} \in \overline{\mathscr{T}}\left(\Omega_{1}, \Omega_{2}\right)$; on the other hand the following result is easily verified.

Proposition 15. $\zeta_{V}^{s}=\zeta_{V^{s}}$ with

$$
V^{s}(t, .):=-D T_{s}^{-1}(-Z(t)) \cdot\left(V(t) o T_{s}(Z(t))^{-1}\right)-\frac{\partial}{\partial t} T_{s}(-Z(t))
$$


In other words:

$$
\frac{\partial}{\partial t} \zeta+\nabla \zeta \cdot V=0 \text { implies } \frac{\partial}{\partial t}\left(\zeta o T_{s}(Z(t))^{-1}\right)+\nabla\left(\zeta o T_{s}(Z(t))^{-1}\right) \cdot V^{s}=0 .
$$

It can also be verified that the expression 89 for the derivative of the field still holds true so that the variational principle $(90)$ is valid for any functional $\mathscr{J}$ minimized over the lipschitzian connecting family $\mathscr{V}^{1, \infty}\left(\Omega_{1}, \Omega_{2}\right)$. And more generally, without assuming $V$ in $E$ we have:

Proposition 16. Let $(\zeta, V) \in \mathscr{T}^{p, q}\left(\Omega_{1}, \Omega_{2}\right)$, then for all $s>0$ and $Z \in \mathscr{Z}$ we have:

$$
\left(\zeta o T_{s}(Z)^{-1}, V^{s}\right) \in \mathscr{T}^{p, q}\left(\Omega_{1}, \Omega_{2}\right) .
$$

In order to get a differentiable metric, we could consider

$$
\tilde{d}\left(\Omega_{1}, \Omega_{2}\right)=\operatorname{Inf}_{V \in \mathscr{V}\left(\Omega_{1}, \Omega_{2}\right)} \int_{0}^{1}\left(\|V(t)\|_{H_{0}^{1} \cap E_{0}}+\left\|\frac{\partial}{\partial t} V\right\|_{L^{2}(D)}\right) d t .
$$

Equipped with $\tilde{d}, \mathscr{O}_{\Omega_{0}}$ would be a complete metric space but $\tilde{d}$ fails to be a metric because of the triangle axiom. The advantage is that now the associated functional is differentiable with repect to $V$, then we can apply the previous variational principle with transverse vector field $Z$. Let $\bar{V}$ be a minimizer in $\mathscr{V}\left(\Omega_{1}, \Omega_{2}\right)$ for $\tilde{d}\left(\Omega_{1}, \Omega_{2}\right)$. Then $\forall Z \in \mathscr{Z}$ we have:

$$
\int_{0}^{1}\left\{\|V(t)\|^{-1}\left\langle V(t), Z_{t}+[Z, V]\right\rangle+\left|V^{\prime}(t)\right|^{-1}\left(\left(V^{\prime}(t)\left(Z_{t}+Z, V\right)^{\prime}\right)\right)\right\} d t=0,
$$

where $\langle$,$\rangle is the H_{0}^{1}\left(D, R^{N}\right)$ inner product while $(()$,$) is the L^{2}\left(D, R^{N}\right)$ one. In order to recover a differentiable complete metric, we introduce again the constraint on the perimeter as in the beginning and set

$$
\delta_{H^{1}}\left(\Omega_{1}, \Omega_{2}\right)=\operatorname{Inf} f_{V \in \mathscr{V}\left(\Omega_{1}, \Omega_{2}\right)} \int_{0}^{1}\|V(t)\|_{H_{0}^{1} \cap E_{0}} d t .
$$

The optimality condition is: $\forall Z \in \mathscr{Z}$

$$
\begin{gathered}
\text { s.t. } \int_{0}^{1} \int_{\Gamma_{t}} H(t)\left\langle Z(t), n_{t}\right\rangle d \Gamma_{t} d t=0 \\
\int_{0}^{1}\|V(t)\|^{-1}\left\langle V(t), Z_{t}+[Z, V]\right\rangle d t=0 .
\end{gathered}
$$

\subsubsection{Euler Equation for Geodesics}

$$
\begin{aligned}
& \exists c(t), P \text { s.t. } \frac{\partial}{\partial t}\left(\|V(t)\|^{p-2} V(t)\right)+\|V(t)\|^{p-2}\left(D V(t) . V+D^{*} V \cdot V(t)\right) \\
& \quad=\nabla P+c \chi_{\Gamma_{t}} \operatorname{div}_{\Gamma_{t}}\left(n_{t}\right) n_{t} .
\end{aligned}
$$


That is,

$$
\begin{aligned}
& (p-2)\|V\|^{p-4}\left(\left(V, \frac{\partial}{\partial t} V\right)\right)+\|V(t)\|^{p-2}\left(\frac{\partial}{\partial t} V+D V(t) \cdot V+D^{*} V \cdot V(t)\right) \\
& \quad=c \chi_{\Gamma_{t}} \operatorname{div}_{\Gamma_{t}}\left(n_{t}\right) n_{t}
\end{aligned}
$$

which can be written as (with $\bar{V}=\|V\|^{-1} V, \Pi=P-1 / 2|V|^{2}$ ):

$$
\begin{gathered}
\operatorname{div} V=0 \\
\frac{\partial}{\partial t} V+(p-2)\left(\left(\frac{\partial}{\partial t} V, \bar{V}\right)\right) \bar{V}=D V \cdot V=\nabla \Pi+c(t)\|V\|^{2-p} \chi_{\Gamma_{t}} \operatorname{div}_{\Gamma_{t}}\left(n_{t}\right) n_{t} .
\end{gathered}
$$

\section{Variational Formulation for Euler Flow}

As an application of the previous results we give a variational formulation for Euler incompressible flow with tube boundary condition. We consider two non miscible fluids and the tube describes the densities distribution. For shortness in this section we assume $p=2$ and we consider the quadratic situation with divergence-free vector fields. Then we consider the Hilbert space

$$
H=\left\{V \in L^{2}\left(I \times D, R^{N}\right) \text { s.t. } \operatorname{div} V=0, V . n_{D}=0\right\} .
$$

We consider any Banach space $E_{1} \subset L^{1}\left(D, R^{N}\right)$ with continuous and compact inclusion mapping.

Examples are $E_{1}=B V\left(D, R^{N}\right)$ or $E_{1}=W^{\varepsilon, p}\left(D, R^{N}\right)$, for $\varepsilon<1 / p, 1 \leq p<\infty$, which is, for $p=2$, the Hilbert space $E_{1}=H^{\varepsilon}\left(D, R^{N}\right)$, for $\varepsilon<1 / 2$.

The set of tubes under consideration is then

$$
\begin{aligned}
& \mathscr{T}=\left\{(\zeta, V) \in L^{2}(I \times D) \times H, \text { s.t. } \zeta=\zeta^{2},\right. \\
&\left.\nabla \zeta \in L^{1}\left(I, E_{1}\right), \zeta_{t}+\nabla \zeta . V=0, \zeta(\tau)=\chi_{\Omega_{1}}\right\} .
\end{aligned}
$$

Notice that the convection equation implies that as $\zeta_{t}=\operatorname{div}(-\zeta V)$, then we get:

$$
\zeta \in C^{0}\left(I, W^{-1,1}\left(D, R^{N}\right)\right) .
$$

Proposition 17. The set $\mathscr{T}$ of tubes is non empty.

\subsection{Tube-Variational Principle}

We introduce the optimal control view point: the state equation will be the convection equation 102 while we shall minimize a "Tube-Energy" cost functional governed by this equation. The regularizing term is a surface tension-like term. As in the previous sections this term will be needed in order to make use of the previous parabolic compactness of tubes. Indeed we shall introduce a kind of "density" perimeter $\theta_{h}$ associated with $L^{1}\left(I, H^{\varepsilon}(D)\right)$ norm of the tube $\zeta$, which turns to be differentiable under smooth transverse fields perturbations $\zeta_{s}$. 


\subsubsection{Given Initial Domain $\Omega_{0} \subset D$}

Being given $\alpha>0, \beta>0$, we consider the following Tube-Energy functional:

$$
\begin{aligned}
\mathscr{E}(\zeta, V) & =1 / 2 \int_{0}^{\tau} \int_{D}(\alpha \zeta(t, x)+\beta)|V(t, x)|^{2} d x d t+\sigma \int_{0}^{\tau}\|\zeta(t)\|_{E_{1}} d t \\
& -\int_{0}^{\tau} \int_{D} V_{0}(x) \cdot V(t, x) d x d t .
\end{aligned}
$$

Theorem 1. The functional $\mathscr{E}$ reaches its minimum on the set $\mathscr{T}$ of tubes.

Proof. We consider a minimizing sequence $\left(\zeta_{n}, V_{n}\right) \in \mathscr{T}$. There exist subsequences such that $V_{n} \rightarrow V$, weakly in $L^{2}(I \times D)$ and $\zeta_{n} \rightarrow \zeta$ strongly in $L^{1}(I \times D)$. Effectively as $\left(\zeta_{n}\right)_{t}=\operatorname{div}\left(-\zeta_{n} V_{n}\right)$, we have:

$$
\left\|\zeta_{n}\right\|_{L^{1}\left(I, E_{1}\right)} \leq M_{1}, \quad\left\|\left(\zeta_{n}\right)_{t}\right\|_{L^{2}\left(I, W^{-1,1}\left(D, R^{N}\right)\right)} \leq M_{2} .
$$

The conclusion derives from the compacity result. From this strong $L^{1}$ convergence we derive that $\zeta^{2}=\zeta$. We consider the weak formulation for the convection problem (102):

$$
\begin{gathered}
\forall \psi \in C^{1}\left(I \times \bar{D}, R^{N}\right), \psi(0, .)=0, \\
\int_{0}^{\tau} \int_{D} \zeta_{n}\left(-\psi_{t}-\nabla \psi \cdot V_{n}\right) d x d t=-\int_{\Omega_{1}} \psi(0, x) d x,
\end{gathered}
$$

in which we can pass to the limit and conclude that $(\zeta, V) \in \mathscr{T}$. Moreover, the element $(\zeta, V)$ is classically a minimizer as the three terms are weakly lower semi- continuous, respectively for each weak topology.

\subsection{Euler Equation Solved by the Minimizer}

In order to analyze the necessary conditions associated with any minimizer of $\mathscr{E}$ over the set $\mathscr{T}$ we introduce transverse transformations of the tube.

\subsubsection{Transverse Field}

Let us consider a perturbation parameter $s \geq 0$ and any smooth horizontal non autonomous vector field over $R^{N+1}$ ( $s$ being the evolution parameter for a dynamic in $\left.R^{N+1}\right)$

$$
\mathscr{Z}(s, t, x)=(0, z(s, t, x)) \in R_{r} \times R^{N},
$$

such that $\mathscr{Z}(s, 0, x)=0$.

For any element $(\zeta, V) \in \mathscr{T}$ we consider the perturbed tube $\left(\zeta^{s}, V^{s}\right)$, where:

$$
\begin{gathered}
\left.\zeta^{s}(t, x):=\zeta o T_{s}\left(\mathscr{Z}^{t}\right)(x)\right)^{-1} \\
V^{s}(t, x)=\left(D\left(T_{s}\left(\mathscr{Z}^{t}\right)^{-1}\right)^{-1} \cdot\left(V(t) o T_{s}\left(\mathscr{Z}^{t}\right)^{-1}-\frac{\partial}{\partial t}\left(T_{s}\left(\mathscr{Z}^{t}\right)^{-1}\right)\right) .\right.
\end{gathered}
$$

Indeed we can show

Proposition 18. $\forall(\zeta, V) \in \mathscr{T}, \forall \mathscr{Z}$, the previously defined elements $\left(\zeta^{s}, V^{s}\right) \in \mathscr{T}$. 


\subsubsection{Transverse Derivative}

Assume that $\operatorname{div}_{x} \mathscr{Z}^{t}=0$, then:

$$
\int_{D}\left(\alpha \zeta^{s}(t, x)+\beta\right)\left|V^{s}(t, x)\right|^{2} d x=\int_{D}(\alpha \zeta(t, x)+\beta)\left|V^{s}(t) o T_{s}\left(\mathscr{Z}^{t}\right)(x)\right|^{2} d x
$$

So that the optimality of the element $(\zeta, V)$ writes:

$$
1 / s\left(\mathscr{E}\left(\zeta^{s}, V^{s} o T_{s}\right)-\mathscr{E}(\zeta, V)\right) \geq 0 .
$$

Now the following quotient has a strong limit in $L^{2}(I \times D)$ :

$$
\begin{aligned}
& \frac{V^{s} o T_{s}-V}{s}=\frac{d}{d s}\left[V^{s} o T_{s}\left(\mathscr{Z}^{t}\right)\right]_{s=0} \\
& =\frac{d}{d s}\left[\left(D\left(T_{s}\left(\mathscr{Z}^{t}\right)^{-1}\right)^{-1} \cdot\left(V(t)-\frac{\partial}{\partial t}\left(T_{s}\left(\mathscr{Z}^{t}\right)^{-1}\right) o T_{s}\left(\mathscr{Z}^{t}\right)\right)\right]_{s=0}\right. \\
& =\frac{d}{d s}\left[\left(D\left(T_{s}\left(\mathscr{Z}^{t}\right) o T_{s}\left(\mathscr{Z}^{t}\right)^{-1} \cdot\left(V(t)-\frac{\partial}{\partial t}\left(T_{s}\left(\mathscr{Z}^{t}\right)^{-1}\right) o T_{s}\left(\mathscr{Z}^{t}\right)\right)\right]_{s=0}\right.\right. \\
& =\frac{\partial}{\partial t} Z(t)+D Z(t) \cdot V(t) \in L^{2}\left(I \times D, R^{N}\right),
\end{aligned}
$$

where we always denote $Z(t)(x)=Z(t, x):=\mathscr{Z}^{t}(0, x)$ (that is at $s=0$ ). Indeed we know that if $V$ was smoother, say $V \in L^{2}\left(H^{1}(\Omega)\right)$, we would have:

$$
\frac{\partial}{\partial s}\left[V^{s}\right]_{s=0}=Z_{t}+[Z(t), V(t)]:=H_{V} \cdot Z
$$

where the Lie bracket is $[Z, V]=D Z . V-D V . Z$, so we would get the previous expression for the derivative of $V^{s} o T_{s}\left(\mathscr{Z}^{t}\right)$, as $\left(V^{s} o T_{s}\right)_{s}=\left(V^{s}\right)_{s}+D V^{s} . D Z(t)$.

\subsection{Necessary Condition}

\subsubsection{Quadratic Term of $\mathscr{E}$}

The quadratic term may be decomposed as follows:

$$
\begin{aligned}
\int_{0}^{\tau} & \int_{D}\left(\left(\alpha \zeta^{s}+\beta\right)\left|V^{s}\right|^{2}-(\alpha \zeta+\beta)|V|^{2}\right) / s d x d t \\
& =\int_{0}^{\tau} \int_{D}\left((\alpha \zeta+\beta)\left(\left|V^{s} o T_{s}\right|^{2}-|V|^{2}\right) / s d x d t\right. \\
& =\int_{0}^{\tau} \int_{D}\left((\alpha \zeta+\beta)\left(V^{s} o T_{s}+V\right)\left(V^{s} o T_{s}-V\right) / s d x d t\right. \\
& \rightarrow 2 \int_{0}^{\tau} \int_{D}\left((\alpha \zeta+\beta) V \cdot\left(\frac{\partial}{\partial t} Z(t)+D Z(t) \cdot V(t)\right) d x d t\right. \\
& =-2\left\langle\frac{\partial}{\partial t}((\alpha \zeta+\beta) V)+{ }^{\prime} D((\alpha \zeta+\beta) V) \cdot V^{\prime}, Z\right\rangle_{\mathscr{D}^{\prime} \times \mathscr{D}} \\
& +\int_{D}\left(\alpha \chi_{\Omega_{\tau}}+\beta\right) V(\tau) \cdot Z(\tau) d x-\int_{D}\left(\alpha \chi_{\Omega_{0}}+\beta\right) V(0) \cdot Z(0) d x,
\end{aligned}
$$

where

$$
" D((\alpha \zeta+\beta) V) \cdot V_{i},=\partial_{j}\left((\alpha \zeta+\beta) V_{i} V_{j}\right) \in W^{-1,1}(D) .
$$

In fact we shall consider $Z$ such that $Z(\tau,)=$.0 over $D$. 


\subsubsection{The Linear Term}

Let $V_{0}$ be any given element in $R^{N}$. We have:

$$
\begin{aligned}
\int_{0}^{\tau} & \int_{D} V_{0} \cdot\left(V^{s}(t, x)-V(t, x)\right) / s d x d t \\
& =\int_{0}^{\tau} \int_{D} V_{0} \cdot\left(V^{s}(t) o T_{s}\left(\mathscr{Z}^{t}\right)(x)-V(t, x)\right) / s d x d t \\
& +\int_{0}^{\tau} \int_{D} V_{0} \cdot\left(V^{s}(t) o T_{s}\left(\mathscr{Z}^{t}\right)(x)-V(t, x)\right) / s d x d t \\
& \rightarrow \int_{0}^{\tau} \int_{D} V_{0} \cdot\left(Z_{t}(t, x)+D Z(t, x) \cdot V(t, x)\right) d x d t \\
& =\int_{0}^{\tau} \frac{\partial}{\partial t}\left(\int_{D} V_{0} \cdot Z(t, x) d x\right) d t=\int_{D} V_{0} \cdot Z(\tau, x) d x-\int_{D} V_{0} \cdot Z(0, x) d x .
\end{aligned}
$$

\subsection{3 "Perimeter" Term in $\mathscr{E}$}

Assume formally that the minimizer element $\zeta$ is smooth enough, so that with the choice $E_{1}=B V\left(D, R^{N}\right)$ we have the surface tension term in the classical form:

$$
\sigma \int_{0}^{\tau}\|\nabla \zeta\|_{M^{1}\left(D, R^{N}\right)} d t=\sigma \int_{0}^{\tau} P_{D}\left(\Omega_{t}\right) d t .
$$

We would obtain as derivative with respect to $s$ :

$$
\sigma \int_{0}^{\tau} \int_{\Gamma_{t}} \Delta b_{\Omega_{t}}\left\langle Z(t), n_{t}\right\rangle d \Gamma_{t} d t
$$

In the interesting case where $E_{1}=W^{\varepsilon, p}(D)$ we introduce the term, for any given "small" $h>0$ :

$$
\theta_{h}(\zeta):=\int_{0}^{\tau}\left(\int_{D \times D} \rho_{h}(\|x-y\|) \frac{|\zeta(x)-\zeta(y)|^{p}}{|| x-y||^{N+\varepsilon p}} d x d y\right) d t,
$$

where $\rho_{h}$ is any smooth positive function such that $\rho(z)=0$ for $|z| \geq 2 h$, and $\rho(z)=1$ for $|z| \leq h$.

As a result we have

\section{Lemma 3}

$$
\int_{0}^{\tau}\|\zeta(t)\|_{W^{\varepsilon, p}(D)} d t \leq \tau\left(\text { meas }(D)+\frac{1}{h^{N+\varepsilon p}} \operatorname{meas}(D)^{2}\right)+\theta_{h}(\zeta) .
$$

So that it is enough to choose the surface tension term in the form $\sigma \theta_{h}(\zeta)$. This term turns to be always differentiable with respect to the transverse perturbations as follows:

$$
\begin{aligned}
& \theta_{h}\left(\zeta o T_{s}(\mathscr{Z})^{-1}\right) \\
& =\int_{0}^{\tau} \int_{D \times D} \rho_{h}\left(\left\|T_{s}(\mathscr{Z})(x)-T_{s}(\mathscr{Z})(y)\right\|\right) \frac{|\zeta(x)-\zeta(y)|^{p}}{\left\|T_{S}(\mathscr{Z})(x)-T_{S}(\mathscr{Z})(y)\right\|^{N+\varepsilon p}} d x d y d t
\end{aligned}
$$


So that, for a.e. $t$ in $I$ we have (with $\theta_{h}(\zeta)=\int_{0}^{\tau} \theta(\zeta(t)) d t$ )

$$
\begin{aligned}
& \frac{\partial}{\partial s} \theta_{h}\left(\zeta^{s}(t)\right)_{s=0}^{p} \\
& =\int_{D \times D} \rho_{h}(\|x-y\|) \frac{|\zeta(x)-\zeta(y)|^{p}}{\|x-y\|^{N+\varepsilon p+2}}\langle x-y, Z(t, x)-Z(t, y)\rangle d x d y \\
& +\int_{D \times D} \rho_{h}^{\prime}(\| x-y||) \frac{|\zeta(x)-\zeta(y)|^{p}}{\|x-y\|^{N+\varepsilon p}}\langle x-y, Z(t, x)-Z(t, y)\rangle d x d y
\end{aligned}
$$

As $\|x-y\| \leq h$ in the previous integrals, we have:

$$
Z(t, x)-Z(t, y)=D Z(t, x+\delta(t)(y-x)) \cdot(y-x) .
$$

There exists a measure $\mu_{h}(\Gamma(t)$ supported by

$$
\Delta_{h}(\Sigma)=\cup_{0<t<\tau}\{t\} \times\left(\cup_{x \in \partial \Omega_{t}} B(x, h)\right),
$$

such that

$$
<\mu_{h}, Z>=\frac{\partial}{\partial s} \theta_{h}\left(\zeta^{s}(t)\right)_{s=0}^{p} .
$$

In some sense when $h \rightarrow 0$ the measure converges to the mean curvature of the moving boundary $\Gamma_{t}$.

\section{Euler-Convection Problem}

We have

Theorem 2. Let $V_{0}$ be any given element in $R^{N}$. Then any minimizer $(\zeta, V)$ to the functional $\mathscr{E}$ over the family of tubes $\mathscr{T}$ solves the following problem:

$$
\begin{gathered}
\frac{\partial}{\partial t} \zeta+\nabla \zeta . V=0, \zeta(0)=\chi_{\Omega_{0}}, \zeta(\tau)=\chi_{\Omega_{1}}, \\
\exists \Pi \text { s.t. } \frac{\partial}{\partial t}((\alpha \zeta+\beta) V)+D((\alpha \zeta+\beta) V) . V+\nabla \Pi=\mu_{h} .
\end{gathered}
$$

Moreover we have

$$
V(0)=\left(V_{0}+\nabla \theta\right) /(\alpha \zeta(0)+\beta)
$$

\section{References}

1. Ambrosio, L.: Lecture notes on optimal transport problems. In: Colli, P., Rodrigues, J.F. (eds.) Mathematical Aspects of Evolving Interfaces. Lecture Notes in Math., vol. 1812, pp. 1-52. Springer, Berlin (2003)

2. Cannarsa, C., Da Prato, G., Zolésio, J.-P.: The damped wave equation in a moving domain. Journal of Differential Equations 85, 1-16 (1990)

3. Cuer, M., Zolésio, J.-P.: Control of singular problem via differentiation of a min-max. Systems Control Lett. 11(2), 151-158 (1988)

4. Delfour, M.C., Zolésio, J.-P.: Structure of shape derivatives for non smooth domains. Journal of Functional Analysis 104(1), 1-33 (1992) 
5. Delfour, M.C., Zolésio, J.-P.: Shape analysis via oriented distance functions. Journal of Functional Analysis 123(1), 129-201 (1994)

6. Delfour, M.C., Zolésio, J.-P.: Shapes and Geometries. Analysis, Differential Calculus, and Optimization. SIAM, Philadelphia (2001)

7. Delfour, M.C., Zolésio, J.-P.: Oriented distance function and its evolution equation for initial sets with thin boundary. SIAM J. Control Optim. 42(6), 2286-2304 (2004)

8. Desaint, F.R., Zolésio, J.-P.: Manifold derivative in the Laplace-Beltrami equation. Journal of Functional Analysis 151(1), 234-269 (1997)

9. Dziri, R., Zolésio, J.-P.: Dynamical shape control in non-cylindrical Navier-Stokes equations. J. convex analysis 6(2), 293-318 (1999)

10. Dziri, R., Zolésio, J.-P.: Dynamical shape control in non-cylindrical hydrodynamics. Inverse Problem 15(1), 113-122 (1999)

11. Dziri, R., Zolésio, J.-P.: Tube derivative of non-cylindrical shape functionals and variational formulations. In: Glowinski, R., Zolésio, J.-P. (eds.) Free and Moving Boundaries: Analysis, Simulation and Control. Lecture Notes in Pure and Applied Mathematics, vol. 252. Chapman $\&$ Hall/CRC (2007)

12. Kawohl, B., Pironneau, O., Tartar, L., Zolésio, J.-P.: Optimal shape design. Lecture Notes in Mathematics, vol. 1740. Springer, Heidelberg (2000)

13. Moubachir, M., Zolésio, J.-P.: Moving shape analysis and control: application to fluid structure interaction. Pure and Applied Mathematics series. CRC, Boca Raton (2006)

14. Da Prato, G., Zolésio, J.-P.: Dynamical programming for non cylindrical parabolic equation. Sys. Control Lett. 11 (1988)

15. Da Prato, G., Zolésio, J.-P.: Existence and control for wave equation in moving domain. In: Stabilization of Flexible Structures. LNCIS, vol. 147, pp. 167-190. Springer, Heidelberg (1990)

16. Sokolowski, J., Zolésio, J.-P.: Introduction to Shape Optimization: Shape Sensitivity Analysis. Springer Series in Computational Mathematics, vol. 10. Springer, Berlin (1992)

17. Zolésio, J.-P.: Introduction to shape optimization and free boundary problems. In: Delfour, M.C. (ed.) Shape Optimization and Free Boundaries. NATO ASI, Series C: Mathematical and Physical Sciences, vol. 380, pp. 397-457 (1992)

18. Zolésio, J.-P.: Shape differential with non smooth field. In: Borggard, J., Burns, J., Cliff, E., Schreck, S. (eds.) Computational Methods for Optimal Design and Control. Progress in Systems and Control Theory, vol. 24, pp. 426-460. Birkhauser, Basel (1998)

19. Zolésio, J.-P.: Variational principle in the Euler flow. In: Leugering, G. (ed.) Proceedings of the IFIP-WG7.2 Conference, Chemnitz. Int. Series of Num. Math., vol. 133 (1999)

20. Zolésio, J.-P.: Weak set evolution and variational applications. In: Shape Optimization and Optimal Design. Lecture Notes in Pure and Applied Mathematics, vol. 216, pp. 415-442. Marcel Dekker, N.Y. (2001)

21. J.-P. Zolésio: Set Weak Evolution and Transverse Field, Variational Applications and Shape Differential Equation INRIA report RR-464 (2002), http: //www-sop.inria.fr/rapports/sophia/RR-464

22. Zolésio, J.-P.: Shape topology by tube geodesic. In: Information Processing: Recent Mathematical Advances in Optimization and Control, pp. 185-204. Presses de l'Ecole des Mines de Paris (2004)

23. Zolésio, J.-P.: Control of moving domains, shape stabilization and variational tube formulations. International Series of Numerical Mathematics, vol. 155, pp. 329-382. Birkhauser Verlag, Basel (2007)

24. Zolésio, J.-P.: Tubes analysis. In: Glowinski, R., Zolésio, J.-P. (eds.) Free and Moving Boundaries: Analysis, Simulation and Control. Lecture Notes in Pure and Applied Mathematics, vol. 252. Chapman \& Hall/CRC (2007) 